\title{
Observations and Models of Coronal Heating
}

\author{
F. Malara
}

Dipartimento di Fisica, Università della Calabria, via P. Bucci, 87036 Rende (CS), Italy

Istituto Nazionale per la Fisica della Materia, Unità di Cosenza, via $P$. Bucci, 87036 Rende (CS), Italy

M. Velli

Dipartimento di Astronomia e Scienza dello Spazio, Università di Firenze, largo E. Fermi, Firenze, Italy

Istituto Nazionale per la Fisica della Materia, Unità di Pisa, Pz Torricelli 2, 56100 Pisa, Italy

\begin{abstract}
Energy release in the solar Corona is characterized by a sequence of space and time localized events, whose intensity follows powerlaw distributions. In quiet Sun regions, small energy events, possibly under the detection threshold, dominate, thus supporting the "nanoflare" scenario of coronal heating. Two complementar models of heating are discussed, in connection with the above observational features. The first model is based on Alfvénic wavepackets dissipation in 3D force-free magnetic fields; the presence of regions of chaoticity of magnetic lines allows for a fast wave dissipation, within a fraction of a solar radius. The second model describes a MHD turbulence in low- $\beta$ plasma, in which magnetic energy is continuously furnished by slow photospheric motions. Energy release events corresponds dissipation of current sheets, often associated with magnetic reconnection. The resulting distribution of dissipated power follows a power law, similar to observations.
\end{abstract}

\section{Introduction}

The nature of energy release in the solar Corona is still a substantially unresolved problem; though different scenarios have been proposed to explain the heating of the corona, they all rely on the transfer, storage and dissipation of the mechanical energy present in photospheric motion; the magnetic field is thought to play a fundamental role in all three steps. This fact led to the idea that coronal activity in its various aspects, including the heating of the plasma up to the observed values of the order of $T \sim 10^{6}$ degrees, may be due to dissipation of magnetic energy. Different energy input mechanisms have been envisaged, which can be roughly divided in two types, according to the associated characteristic time scale $\tau_{i n}$. 
In "quasi-static" mechanisms $\tau_{i n} \gg \tau_{A}$, where $\tau_{A}$ is the Alfvén transit time, i.e., the typical time necessary for an Alfvén wave to cross a coronal structure. Assuming a magnetic field $B_{0} \sim 100 \mathrm{G}$, a density $n \sim 10^{9} \mathrm{~cm}^{-3}$, the Alfvén transit time for a loop of length $L \sim 10^{10} \mathrm{~cm}$ would be $\tau_{A} \sim 20 \mathrm{~s}$. In this case, magnetic energy is slowly stored in the coronal field, while the structure moves through a sequence of quasi-equilibrium states. Eventually, the stored energy is rapidly released in a burst of activity. Parker (1972) first suggested that coronal heating could result from the energy flux associated with the tangling of coronal field lines by photospheric motions.

In wave-based mechanisms energy is continuously injected in the Corona by a flux of waves, whose energy is somehow dissipated in the coronal plasma (see, e.g., a review by Malara \& Velli 1994); in this case $\tau_{\text {in }} \lesssim \tau_{A}$. The input energy flux $\varepsilon$ necessary to balance energy losses at a typical coronal temperature has been estimated as $\varepsilon \sim 5 \times 10^{5} \mathrm{erg} \mathrm{cm}^{-2} \mathrm{~s}^{-1}$ for a coronal hole, up to $\varepsilon \sim 10^{7} \mathrm{erg}$ $\mathrm{cm}^{-2} \mathrm{~s}^{-1}$ in an active region (Withbroe 1988; Krucker \& Benz 1998). The corresponding flux of Alfvén waves is given by $S=\rho(\delta v)^{2} c_{A} / 2$. Nonthermal motions $\delta v \sim 50 \mathrm{~km} \mathrm{~s}^{-1}$ (Acton et al. 1981) have been detected in the Corona; if this velocity is interpreted as due to wave motions, using the above values for density and magnetic field, we obtain $S \simeq 1.5 \times 10^{7} \mathrm{erg} \mathrm{cm}^{-2} \mathrm{~s}^{-1}$. Then, the energy flux supplied by Alfvén waves would be enough to heat the Corona at the observed temperature. A more difficult point is how to dissipate energy, due to the extreme smallness of the relevant dissipative coefficients in the coronal plasma. In such conditions, dissipation requires an efficient generation of small scales structures. In wave-based theories small scales are generated in consequence of the inhomogeneity of the background structure, while in quasi-static mechanisms magnetic reconnection is responsible for magnetic energy dissipation.

Observations have shown that the energy release in Corona is always characterized by a series of impulsive events, which cover a large energy range. This fact represents a further constraint for coronal heating models, which should be able to reproduce such a feature. For instance, the so-called SOC (self-organized criticality) models reproduce several statistical properties of the energy release events, namely, the distribution of peak intensity, total energy and duration (Lu \& Hamilton,1991). However, these models are not completely satisfactory because they try to modelize only reconnection and magnetic energy input, but the internal dynamics of the plasma is neglected.

Here we will briefly review some recent observational result concerning the statistics of energetic events in the Corona. Next, we will discuss two heating models, based on wave dissipation and on quasi-static energy input, respectively.

\section{Observational Results}

Parker (1988) first introduced the idea that coronal heating is due to the effect of a multitude of small events, each releasing a small amount of energy. These events, which Parker called "nanoflare" ( 10 $\left.{ }^{24} \mathrm{erg}\right)$, should essentially be due to localized magnetic reconnection. The observed energy release in the Corona is actually impulsive, i.e., it appears as a sequence of single events. The energy associated to each event has been estimated to range from $\sim 10^{32}$ erg (corresponding to a large flare) down to $\sim 10^{23}-10^{24} \mathrm{erg}$. The lower limit is probably 
not a physical property, but it is due to instrumental limitations. Hard X-ray spikes have been observed (Lin et al. 1984) in the energy range $E \sim 10^{24}$. $10^{27} \mathrm{erg}$; these spikes becomes more numerous when decreasing the energy $E$. Porter et al. (1987) reported localized brightenings throughout the magnetic network; this indicates that single events are localized not only temporally, but also spatially.

Impulsive energy release events can be characterized giving the distribution of energy, or of some other observational parameter giving a measure of the intensity of a single event. Such distributions indicate that the less energetic events are the more numerous; specifically, they often follow a power law. Lu \& Hamilton (1991) calculated the distribution of peak photon flux in hard Xray flares, finding that it can be well approximated by a power law with index $\delta \simeq 1$. The value of the index $\delta$ is important in order to determine whether the global energy release is mostly due to small energy or large energy events. For instance, indicating by $f(E) \propto E^{-\delta}$ the distribution of energy released in a given time, the total energy in the range $\left[E_{\text {min }}, E_{\text {max }}\right]$ is given by

$$
E_{t o t}=\int_{E_{\min }}^{E_{\max }} E f(E) d E \propto \begin{cases}\frac{1}{(2-\delta) E_{\max }^{\delta-2}}, & \text { if } \delta<2 \\ \frac{1}{(\delta-2) E_{\min }^{\delta-2}}, & \text { if } \delta>2\end{cases}
$$

Thus, if the energy distribution $f(E)$ is quite flat $(\delta<2)$, then small energy events are not relevant, and heating should mainly due to the most energetic events. Conversely, if $f(E)$ is steep enough $(\delta>2)$ then coronal heating is sustained by small energy events; this would correspond to the Parker's nanoflares scenario. In such a case, a fraction of such heating events could have energies too small to be detected by observations.

In active regions, energy release seems to be dominated by large energy events. Crosby et al. (1993) and Shimizu (1995) calculated power-law distributions with an index $\delta \simeq 1.5-1.6$. The situation is different in the quiet Corona: using data from the EUV telescope on SOHO, Krucker \& Benz (1998) calculated the energy distribution for the events in a low energy range $\left(E \simeq 8 \times 10^{24}-3 \times 10^{26}\right.$ erg). They found a power-law distribution with an index $\delta=2.3-2.6$. The corresponding power per unit surface is $\varepsilon \sim 7 \times 10^{4} \mathrm{erg} \mathrm{cm}^{-2} \mathrm{~s}^{-1}$, which has been estimated as $\sim 16 \%$ of the energy flux necessary to sustain the quiet Corona. The remaining fraction of power could be ascribed to events at energies below the lower limit of the considered range. Similar results have been found by Parnell \& Jupp (2000), who used data from TRACE relative to quiet Corona regions. Employing an elaborate technique, these authors found power-law energy distributions, with an index $\delta$ in the range 2.04-2.5, for events at energies $z 3 \times 10^{23}$ erg. Though the values found for the index $\delta$ of power-law distributions are somehow model-dependent, it seems that lowest energy events should give the dominant contribution to the global heating, at least in the quiet Corona. Models of coronal heating should account for these observational features, namely, the impulsive nature of the energy release, and, possibly, power-law distributions of energy releasing events. 


\section{Dissipation of Alfvén Waves in 3D Magnetic Structures}

Some direct observation of waves in the coronal plasma have been recently reported: De Forest \& Gurman (1988) detected coherent wave trains in polar plumes, which have been interpreted as slow magnetosonic waves; Thompson et al. (1999) and Nakariakov et al. (1999) observed propagating features, related to large flares. No direct measures of Alfvén waves in the Corona exists; however, such waves represent the main component of solar wind fluctuations (e.g., Belcher \& Davis 1971), which probably are originated in the Corona.

A key point in wave-based theories of coronal heating is how to dissipate the wave energy, i.e., how to generate small scales in fluctuations. This can be achieved when the background medium is inhomogeneous. An Alfvén wave, propagating in a region where the Alfvén speed $\mathbf{c}_{A}=\mathbf{B} /(4 \pi \rho)^{1 / 2}$ varies in a direction transverse to the wave propagation, undergoes phase-mixing (Heyvaerts \& Priest 1983): differences in the phase speed stretch the wavepacket, and the wavevector increases linearly in time $k_{\perp} \sim \gamma t$. The corresponding dissipation time follows the scaling law

$$
t_{d}^{p m} \propto S^{1 / 3}
$$

where $S$ is the Reynolds/Lundquist number. This phenomenon have been studied in various circumstances for 1D equilibrium structures (Lee \& Roberts 1986, Califano et al 1990; Malara et al 1992, 1996). The scaling law (2) holds also for $2 \mathrm{D}$ equilibria, if regular surfaces with $c_{A}=$ const can be defined. However, phase-mixing could take a quite long time to dissipate waves; assuming, for instance, $c_{A} \sim 10^{3} \mathrm{~km} \mathrm{~s}^{-1}$, the equilibrium scale length $a \sim 10^{3} \mathrm{~km}$, the wavelength $\lambda_{\|} \sim(1-10) a$, and $S \sim 10^{8}-10^{11}$, we find a dissipation length $l_{d}=c_{A} t_{d} \sim(2-100) R_{\odot}$ (Malara et al. 1996). Over such distances, variations of the equilibrium in the 3rd direction are no longer negligible.

The situation can drastically change in 3D equilibria. In such a case, regions where magnetic lines are chaotic can exist. In the Corona, these structures might results from photospheric motions, which twist and braid magnetic lines (Berger 1991). In a chaotic region, neighbouring magnetic lines exponentially diverge. Thus, an Alfvénic packet, which locally follows magnetic lines, is stretched, and small scales are formed exponentially in time $k \propto \exp (\gamma t)$. The scaling law for dissipation time is (Similon \& Sudan 1989):

$$
t_{d}^{3 D} \propto \log S
$$

For large values of $S$, the dissipation time can be much shorter than in 1D or 2D configurations. Petkaki et al. (1998) built up a model to study this phenomenon. This model describes the propagation of small amplitude waves in an incompressible plasma, assuming a perturbation scale length $\delta \ll a$. A WKB expansion allows to obtain the following equations

$$
\frac{d x_{j}}{d t}=c_{a j}(\mathbf{x}) \quad ; \quad \frac{d k_{j}}{d t}=-k_{n} \frac{\partial c_{a n}}{\partial x_{j}} ; \frac{d e}{d t}=-\frac{2 k^{2}}{S} e
$$

describing the time evolution of packet position $\mathbf{x}$, wavevector $\mathbf{k}$, and energy $e$. Phase-mixing and 3D effects can co-exist in a given equilibrium field; a simple case describing this situation is the so-called $A B C$ magnetic field, which 
represents a force-free equilibrium field. In this field, there are regions where magnetic lines are chaotic, along with regions with quasi-regular magnetic surfaces (ordered regions) (Dombre at al. 1986). Malara et al. (2000) considered an Alfvénic perturbation formed by a large number of packets, and followed the time evolution of each packet in the ABC field by integrating the equations (4). The results show that in this simple case the packets can be divided in two distinct populations, according to the dissipation time of a single packet. The first population, indicated by QDP (quickly dissipated packets) is formed by packets which essentially propagate in the regions where the magnetic lines are chaotic. The average dissipation time $t_{d}^{Q D P}$ of this population turns out to be proportional to $\log S$. Thus, such a population, which is dissipated faster than the other one, follows a 3D dynamics, determined by the chaoticity of the region where it propagates. The second population, indicated by SDP (slowly dissipated packets) is formed by packets propagating in ordered regions. The corresponding dissipation time follows the scaling law $t_{d}^{S D P} \propto S^{1 / 3}$. For these packets the dominant dynamical mechanism is phase-mixing, as for a $2 \mathrm{D}$ configurations. Then, in the ABC magnetic field both mechanisms are present, but in spatially distinct regions. The time evolution of the whole Alfvénic perturbation results from the superposition of these two behaviors, whose differences increase with increasing $S$. The fraction of the total energy which is dissipated by the fastest mechanism depends on how wide are the chaotic regions; the maximum value attained is $E_{Q D P} / E_{0} \simeq 30 \%$.

Malara et al. (2000) considered also a more complex magnetic equilibrium, denoted as "multicomponent force-free field", which is defined by

$$
\mathbf{B}^{(0)}(x, y, z)=\mathbf{B}^{2 D}(x, y)+\varepsilon \mathbf{B}^{3 D}(x, y, z)
$$

where $\mathbf{B}^{2 D}(x, y)=(\cos y) \mathbf{e}_{x}+(\sin x) \mathbf{e}_{y}+(\sin y+\cos x) \mathbf{e}_{z}$ represents $2 \mathrm{D}$ parallel flux tubes, and

$$
\mathbf{B}^{3 D}(x, y, z)=\sum_{i=1}^{N} A^{(i)}\left[\sin \left(\mathbf{e}_{3}^{(i)} \cdot \mathbf{x}\right) \mathbf{e}_{1}^{(i)}+\cos \left(\mathbf{e}_{3}^{(i)} \cdot \mathbf{x}\right) \mathbf{e}_{2}^{(i)}\right]
$$

represents a $3 \mathrm{D}$ distorsion. Increasing its amplitude $\varepsilon$, starting from $\varepsilon=0$, the geometry of the magnetic field $\mathbf{B}^{(0)}$ goes from being purely $2 \mathrm{D}$ to fully $3 \mathrm{D}$. The evolution of an Alfvénic perturbation has been followed by integrating the equations (4) for a large number of packets, but in this case it is no longer possible to distinguish between two populations. The dissipation time $t_{d}$ follows either the scaling law (2) or (3): for large Reynolds numbers (above a threshold $S_{t h r}(\varepsilon)$ ) it is found $t_{d} \propto \log S$ (3D regime), while $t_{d} \propto S^{1 / 3}$ for $S<S_{t h r}(\varepsilon)$ (phase-mixing regime). Due to the more complex geometry of the magnetic field (5), during its time evolution every packet, "visits" both chaotic and ordered regions, following both a 3D dynamics and phase-mixing. Then, the dissipation of the whole perturbations is determined by the fastest of these two mechanisms, which is always $3 \mathrm{D}$ dissipation, if $S$ is large enough. With decreasing the amplitude $\varepsilon$ of the $3 \mathrm{D}$ component, the chaotic regions become smaller, thus, the threshold $S_{t h r}(\varepsilon)$ separating the two regimes increases. However, even considering very small 3D perturbations, like $\varepsilon=0.05$, the system is in the $3 \mathrm{D}$ regime as soon as $S>10^{8}-10^{9}$. In the coronal plasma where $S$ could attain $10^{12}$, including a 
small 3D perturbation can reduce $t_{d}$ by a large factor with respect to the purely 2D configuration: for instance, using $\varepsilon=0.1, S=10^{12}$ and the above values for $a$ and $c_{A}$, the dissipation length is only $l_{d} \sim 0.4 R_{\odot}$, which is much shorter than the estimation made in the phase-mixing case.

Besides estimating the efficiency of dissipation, it is interesting to study how dissipation behaves when the system is continuously feed by energy. This is achieved by injecting new packets inside the magnetic structure, for instance, uniformly distributed in time. Each packet will evolve differently, according to its trajectory in the equilibrium structure, and it will be dissipated in a different time. The resulting dissipated power $w(t)$ is a sequence of bursts with variable amplitudes. The peak distribution of $w(t)$ depends on the parameters of the model; dissipation becomes more intermittent close to the border between the phase-mixing and the $3 \mathrm{D}$ regimes. In the latter case, packets follows the 3D dynamics only occasionally; when this happens, stronger isolated dissipation peaks form. We now come to a discussion of the Parker scenario. Remark that what we have just said about waves applies also to fluctuations generated by the micro-flares of Parker, and their evolution is followed below within the framework of MHD.

\section{Numerical simulations of the Parker scenario}

Parker was the first to develop a scenario in which the energy dissipation responsible for the heating of the corona occurs in small scale current sheets arising from the dynamics of coronal magnetic field lines attempting to rearrange themselves into an equilibrium situation when subject to random footpoint shuffling. From a quantitative point of view, Parker (1988) derived values of the mean transverse component of the coronal field produced by the external forcing and of the time scale of its production by order-of-magnitude arguments, neglecting the internal dynamics of the system driven by the external forcing. The amount of energy liberated by dissipation, computed with this procedure, is the total energy dissipated averaged over the entire system and over a time long compared with the dynamical time. In reality the evolution of a system governed by MHD equations is highly dynamic, and both the mean dissipation as a function of time as well as the spatial current distribution present an intermittent character (Einaudi et al., 1996, Georgoulis et al, 1998, Dmitruk et al. 1998)

This means that there are many current sheets associated with any observed radiation emission and that in an individual "spatial" dissipative event the amount of energy involved is much smaller than the nanoflare energy estimated by Parker.

With this in mind we now summarize results obtained from very long time simulations of a magnetically forced MHD system, taken to represent the dynamics of a cross section of a coronal structure such as a loop, where the underlying field is essentially a potential one. Because the loop is threaded by a strong axial magnetic field (the potential component), plasma dynamics occurs in transverse planes where the flow is approximately incompressible, while communication from the photosphere across planes occurs via linear propagation of Alfvén waves. In a cartesian approximation (i.e. the loop is straightened out and there are two photospheres), the dynamics of the loop is therefore described 
by the equations of reduced MHD:

$$
\begin{gathered}
\rho\left(\frac{\partial \vec{u}_{\perp}}{\partial t}+\overrightarrow{u_{\perp}} \cdot \vec{\nabla} \vec{u}_{\perp}\right)=-\vec{\nabla}_{\perp}\left(p+\frac{1}{2} b_{\perp}^{2}\right) \\
+\vec{b}_{\perp} \cdot \vec{\nabla} \vec{b}_{\perp}+B_{0} \frac{\partial \vec{b}_{\perp}}{\partial z}+\nu \nabla_{\perp}^{2} \vec{u}_{\perp} \\
\frac{\partial \vec{b}_{\perp}}{\partial t}=\vec{b}_{\perp} \cdot \vec{\nabla} \vec{u}_{\perp}-\vec{u}_{\perp} \cdot \vec{\nabla} \vec{b}_{\perp}+B_{0} \frac{\partial \vec{u}_{\perp}}{\partial z}+\eta \nabla_{\perp}^{2} \vec{b}_{\perp}
\end{gathered}
$$

where $b_{\perp}$ and $\vec{u}_{\perp}$ are the transverse magnetic field and velocities, $z$ is the axial direction, $\rho$ is the mass density, $p$ is the plasma pressure and $\nu, \eta$ are the collisional dissipation coefficients, namely the kinematic viscosity and the resistivity respectively. These equations are valid in the limit of small ratio of kinetic to magnetic pressures, large loop aspect ratio $(\epsilon \equiv l / L<<1, L$ being the length of the loop and $l$ the minor radius of the loop), and small ratio of poloidal to axial magnetic field $\left(b_{\perp} / B_{0}<\epsilon\right)$. The latter condition also ensures incompressibility of plasma motion in the plane. As a consequence the density, considered initially to be uniform, allowing use of the same units for velocity and magnetic fields via the normalization $b \rightarrow b / \sqrt{\rho}$.

To carry out sufficiently long simulations, a further simplification has been considered, whereby the propagation of Alfvén waves along loops was replaced by a stochastic large-scale magnetic forcing function in the induction equation, rendering the system entirely $2 \mathrm{D}$. In other words z-derivatives are written as

$$
\begin{aligned}
& B_{0}\left(\frac{\partial \vec{u}_{\perp}}{\partial z}\right)=\vec{F}_{m}(x, y, t) \\
& B_{0}\left(\frac{\partial \vec{b}_{\perp}}{\partial z}\right)=\vec{F}_{u}(x, y, t)
\end{aligned}
$$

where $\vec{F}_{m}, \vec{F}_{u}$ are unknown forcing functions.

A justification of this approximation, which also illustrates its potential limitations, involves a discussion of Alfvén wave propagation characteristics. Three different time-scales in the RMHD equations, namely propagation-time along the loop, $\tau_{A}=L / B_{0}$, the non-linear timescale $\tau_{\perp}=l / b_{\perp}$, which in the reduced MHD ordering satisfies $\tau_{\perp}>\tau_{A}$, and a typical photospheric time scale $\tau_{p h}$, which is orders of magnitude larger. For times $t \leq \tau_{\perp}$ non-linear terms can be safely neglected: in this situation the magnetic structure which results within the volume is a direct mapping of the photospheric velocity field. For example if the velocity field at one edge of the loop vanishes, and is given at the other by $\vec{u}_{\perp}=\vec{u}_{\perp}^{0}(x, y) \cos \left(\omega_{p h} t\right)$, the linear solution is given by

$$
\begin{aligned}
& \vec{u}_{\perp}=\vec{u}_{\perp}^{0}(x, y) \cos \left(\omega_{p h} t\right) \sin \left(\omega_{p h} z / B_{0}\right) / \sin \left(\omega_{p h} L / B_{0}\right), \\
& \vec{b}_{\perp}=\vec{u}_{\perp}^{0}(x, y) \sin \left(\omega_{p h} t\right) \cos \left(\omega_{p h} z / B_{0}\right) / \sin \left(\omega_{p h} L / B_{0}\right),
\end{aligned}
$$

as long as $\omega$ does not belong to a discrete set of resonant frequencies. Now the photospheric time-scales are long compared to the Alfvén travel time along the loop so that $\sin \left(\omega_{p h} L / B_{0}\right) \simeq \omega_{p h} L / B_{0}, \sin \left(\omega_{p h} z / B_{0}\right) \simeq \omega_{p h} z / B_{0}$. Hence

$$
\vec{u}_{\perp}=\vec{u}_{\perp}^{0}(x, y) \cos \left(\omega_{p h} t\right) z / L,
$$


while the magnetic field is given by

$$
\vec{b}_{\perp}=\vec{u}_{\perp}^{0}(x, y) \sin \left(\omega_{p h} t\right) B_{0} / L \omega_{p h} .
$$

Also, $\sin \left(\omega_{p h} t\right) \simeq \omega_{p h} t$ since $\tau_{p h}$ is by far the longest time, and the linear solution is cut short when $t \simeq \tau_{\perp}$. At that time, therefore, one may estimate that the magnetic energy averaged over the loop length is greater than the kinetic energy averaged over the loop length by a factor $2 \tau_{\perp}^{2} / \tau_{A}^{2}$.

If the two-dimensional plane represents an average over the fastest timescale $\tau_{A}$ or equivalently an average over loop length of the transverse loop dynamics, neglecting kinetic forcing in the momentum equation with respect to the magnetic forcing in the induction equation is therefore a reasonable assumption. Thus, in many respects, such simulations are a realization of Parker's scenario, where the detail of the perpendicular dynamics may be followed without any assumptions on the properties of the resulting current sheets. Note that the transverse dynamics at $t>\tau_{\perp}$ causes the magnetic field structure to depart substantially from the mapping determined by the photospheric flow. As a consequence, the energy input into the system, which is the product of the forcing with the instantaneous field configuration in the loop, is not given solely by the forcing, but is determined by the internal dynamics of the system. This is why the corona must be considered a self-organized system.

Numerical simulations have been carried out for a very long period of time (expressed in terms of the typical non-linear time-scales in the loop cross sections) allowing for a significant statistical sample. The adopted forcing has the functional form

$$
\vec{F}_{m}=\vec{\nabla} \times f_{m} \hat{z}, \quad f_{m}=A_{1}(x, y) \sin ^{2}\left(\frac{\pi t}{2 t^{*}}\right)+A_{2}(x, y) \sin ^{2}\left(\frac{\pi t}{2 t^{*}}+\frac{\pi}{2}\right)
$$

where

$$
A_{i}=\sum_{n m} \alpha_{n m}^{i} \sin \left(k_{n} x+k_{m} y+\zeta_{n m}^{i}\right)
$$

The wavenumber values $k$ used are all those in the range $3 \leq\left(k_{n}^{2}+k_{m}^{2}\right)^{1 / 2} \leq 4$. Notice that the forcing term consists of two "eddies" with a turnover time $2 t^{*}$, which are temporally out of phase. At the end of each $t^{*}$-interval the terms $\alpha_{n m}^{i}$ and $\zeta_{n m}^{i}$ are alternately changed randomly (with a uniform distribution over the intervals $[0,1]$ and $[0,2 \pi]$ respectively) for the eddy of vanishing amplitude. The $\alpha_{n m}^{i}$ are then renormalized so that the spatial average of $A_{i}$ is $\left\langle A_{i}\right\rangle=1$, implying that the rms spatial non-dimensional value for the forcing term $f_{m}$ is also unity $\left(\left\langle f_{m}\right\rangle=1\right)$. The physical units of the model are then fixed in terms of the large-scale magnetic field $B_{0}$, the typical photospheric velocity $u_{p h}$ (in units of $B_{0}$ ), the loop length $L$ and the aspect ratio $1 / \epsilon$.

The units of magnetic field/velocity and length used to render the equations non-dimensional are respectively $b_{0}$ and $l_{\perp}$, which also define the time unit $\tau=l_{\perp} / b_{0}$. From Equ.(14) one sees that

$$
1 \sim \frac{B_{0}}{b_{0}} u_{p h} \frac{\tau}{L}
$$


and eliminating $\tau$ in favor of $b_{0}, l_{\perp}$ gives

$$
b_{0} \sim B_{0}\left(\frac{l_{\perp} u_{p h}}{B_{0} L}\right)^{\frac{1}{2}}
$$

$b_{0}$ is the poloidal Alfvén velocity; it is the only velocity unit directly relevant to the simulations, since the axial field does not appear in the equations. Assuming the photospheric velocity to satisfy $u_{p h} \simeq 0.001 B_{0}$, and a value $1 / \epsilon=10$ for the loop aspect ratio, we obtain from Equation (18)

$$
\frac{b_{0}}{B_{0}} \simeq 0.01 \quad, \quad \tau \simeq 10 \frac{L}{B_{0}} s
$$

If $B_{0} \simeq 1000 \mathrm{~km} / \mathrm{s}$ and $L \simeq 10^{4} \mathrm{~km}, \tau$ turns out to be $\tau \simeq 100 \mathrm{~s}$. Resistivity and viscosity are adapted to the grid and $\tau^{*}=16 \tau$.

The unit for the energy dissipation follows directly from the parameters given above and an average value for the coronal density $\left(10^{9} \mathrm{~cm}^{-3}\right)$ :

$$
\left[\eta J^{2}\right]=4 \pi \rho b_{0}^{2} / \tau
$$

which for a density of $10^{9} \mathrm{~cm}^{-3}$ yields a dissipation unit of $2.110^{-4} \mathrm{erg} / \mathrm{cm}^{3} / \mathrm{s}$. The time evolution of the current dissipation averaged over the whole system $E_{D}=\left\langle\eta J^{2}>\right.$, from a simulation with a $128 \times 128$ resolution was shown in (Georgoulis et al. 1998). The time-series shows strong fluctuations, with an order of magnitude variation of the dissipated energy over short time scales. Intense dissipative events occur well above a continuous active background of small-scale flickering. The quantitative definition of a "dissipative event" allows for some ambiguity, above all if the distribution of such events must be compared with the distribution of "observed" events. In Goergoulis et al. (1998), we made the choice of subtracting from the discrete distribution function of the dissipated power its low energy component, which is well fitted by a $\chi^{2}$-distribution function, and can be interpreted as noise. The measured distribution function starts to deviate significantly from the $\chi^{2}$-fit for $E_{D} \geq E_{n}=1.6$. A dissipative event is then defined to start when the dissipation exceeds $E_{n}$ and to end when it decreases below $E_{n}$. In Georgoulis et al. (1998) 369 discrete events were observed which when plotted, showed a robust power law extending over 2.5 orders of magnitude, with a scaling index $\delta_{T}=-1.32 \pm 0.24$.

The spatial distribution of the fields at different instants shows how the change in the average dissipation is due both to a varying number of current layers and to a different current intensity in each. Collecting a sample of spatial configurations with a sufficient intervening time-lag to ensure a statistically uncorrelated sample allows one to compute histograms of the current intensity. This also displays non-gaussian behaviour, and power laws for the distribution of intensities above noise.

This suggests that in the limit of large magnetic Reynolds numbers, all of the dissipation comes from sheets separating large scale loops, a possibility which is supported by the inverse cascade of the magnetic vector potential in $2 \mathrm{D}$ and reduced MHD. That the dissipation in the sheets is not a purely diffusive process may be seen by examining the structure of magnetic and velocity fields together with currents and vorticity at any given instant, which display quadrupolar 
vorticity structures surrounding current sheets, the typical signature of magnetic reconnection.

In summary, we have seen how an appropriate statistical analysis of a forced MHD system reveals some of the statistical features common to observational properties of small scale energetic events on the Sun, namely power law type distributions in energy/peak-luminosity and duration, and that the building blocks for such behaviour are thin current sheets of variable number and intensity which develop between larger-scale loop like structures. The two-dimensional simulations have been followed up with fully $3 \mathrm{D}$ ones, but for much shorter time-scales. The main differences between the two types of simulations comes from the line-tying effect, namely the presence of wave-propagation in the third direction. On the one-hand, this determines a condition for the onset of the turbulent cascade in the transverse direction requiring stronger gradients in the field. On the other, it also inhibits the inverse cascade of magnetic potential, stopping the configuration from ever reaching a state totally indipendent of the photospheric forcing flow. A detailed discussion of such questions will soon be presented elsewhere (Betta et al. 2001, preprint).

\section{References}

Acton L. W., et al., 1981, ApJ, 224, L137

Belcher, J. W., \& Davis, L., 1971, J. Geophys. Res. 76, 3534

Berger, M. A., 1991, A\&A, 252, 369

Califano, F., Chiuderi, C., \& Einaudi, G., 1990, ApJ, 365, 757

Crosby, N. B., Aschwanden, M. J., \& Dennis, B. R., 1993, Sol. Phys., 39, 155

DeForest, C. E., \& Gurman, J. B., 1988, ApJ, 501, L217

Dmitruk, P., Gomez, D. O., \& DeLuca, E. E., 1998, ApJ, 505, 974

Dombre, T., Frisch, U., Greene, J. M., Hénon, M., Mehr, A., \& Soward, A. M., 1986, J. Fluid Mech., 167, 353

Einaudi, G., Velli, M., Politano, H., \& Pouquet, A., 1996 ApJ, 457, L113

Georgoulis, M., Velli, M., \& Einaudi, G., 1998, ApJ, 497, 957

Heyvaerts, J., \& Priest, E. R., 1983, A\&A, 117, 220

Krucker, S., \& Benz, A. O., 1998, ApJ, 501, 1213

Lee, E. M., \& Roberts, B., 1986, ApJ, 301, 430

Lin, R. P., Schwartz, R. A., Kane, S. R., Pelling, R. M., \& Hurley, K. C., 1984, ApJ, 283, 421

Lu, E. T., \& Hamilton, R. J., 1991, ApJ, 380, L89

Malara, F, Petkaki, P., \& Veltri, P., 2000, ApJ, 533, 523

Malara, F., Primavera, L., \& Veltri, P., 1996, ApJ, 459, 347

Malara, F., \& Velli M., 1994, Wave-based heating mechanisms for the solar corona, in Solar Coronal Structures, proceedings of the IAU Colloquium 144, V. Rusin, P. Heinzel \& J.-C. Vial eds., Veda Publishing

Malara, F., Veltri, P., Chiuderi, C., \& Einaudi, G., 1992, ApJ396, 297 
Nakariakov, V. M., Ofman, L., DeLuca, E. E., Roberts, B., \& Davila J. M., 1999, Science, 285, 862

Parker, E. N., 1972, ApJ, 174, 599

Parker, E. N., 1988, ApJ, 330, 474

Parnell, C. E., \& Jupp. P. E., 2000, ApJ, 529, 554

Petkaki, P., Malara. F., \& Veltri, P., 1998, ApJ, 500, 483

Porter, J. G., et al., 1987, ApJ, 323, 380

Similon, P. L., \& Sudan, R. N., 1989, ApJ, 336, 442

Shimizu, T., 1995, PASJ, 47, 251

Thompson, B. J., et al., 1999, ApJ, 517, L151

Withbroe, G. L., 1988, ApJ, 325, 442 